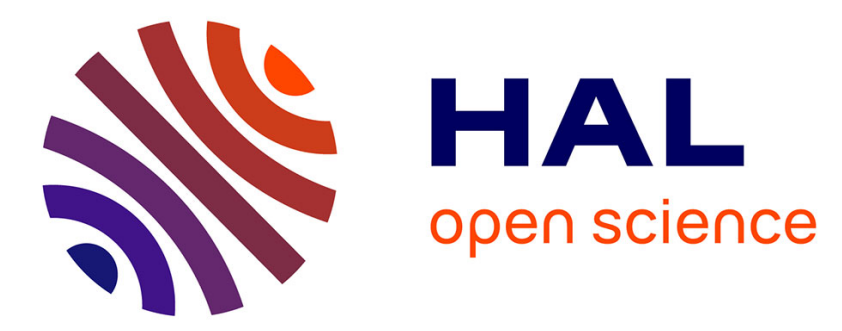

\title{
Collision-Free Formation Tracking of Multi-Agent Systems Under Communication Constraints
}

Syed A Ajwad, Emmanuel Moulay, Michael Defoort, Tomas Menard, Patrick Coirault

\section{- To cite this version:}

Syed A Ajwad, Emmanuel Moulay, Michael Defoort, Tomas Menard, Patrick Coirault. CollisionFree Formation Tracking of Multi-Agent Systems Under Communication Constraints. IEEE Control Systems Letters, 2021, 5 (4), pp.1345-1350. 10.1109/LCSYS.2020.3036809 . hal-03045472

\section{HAL Id: hal-03045472 https://hal.science/hal-03045472}

Submitted on 8 Dec 2020

HAL is a multi-disciplinary open access archive for the deposit and dissemination of scientific research documents, whether they are published or not. The documents may come from teaching and research institutions in France or abroad, or from public or private research centers.
L'archive ouverte pluridisciplinaire HAL, est destinée au dépôt et à la diffusion de documents scientifiques de niveau recherche, publiés ou non, émanant des établissements d'enseignement et de recherche français ou étrangers, des laboratoires publics ou privés. 


\title{
Collision-free formation tracking of multi-agent systems under communication constraints
}

\author{
Syed A. Ajwad, Emmanuel Moulay, Michael Defoort, Tomas Ménard and Patrick Coirault
}

\begin{abstract}
This paper deals with the problem of collisionfree formation tracking of second-order Multi-Agent Systems (MAS) under communication constraints. It is assumed that an agent can only transmit its position to its neighbors at asynchronous and aperiodic discrete time instants. Velocity and acceleration of the agents are not available. Moreover, the dynamics of the leader can be controlled through an external input. Using continuousdiscrete time observers, an output feedback formation controller is designed to drive the agents to a desired formation shape and to ensure that the formation tracks the leader trajectory under directed communication topology. A potential function based mechanism is incorporated into the proposed formation tracking algorithm to avoid collision between agents. The simulation results have shown the efficacy of the proposed algorithm.
\end{abstract}

Index Terms-Artificial potential function, Continuousdiscrete time observer, Formation tracking, Nonuniform and asynchronous sampling

\section{INTRODUCTION}

$\mathbf{F}$ ORMATION patterns produced by natural organisms to deal with different situations have attracted the attention of the research community. For instance, ants generate different patterns to move large chunks of food from a source to their underground nest and some birds fly in a V shape to reduce flying efforts. Other examples of formation producing tasks in nature are bacteria swarming and fish schooling. Motivated by these examples, formation control of Multi-Agent Systems (MAS) has been widely studied in the past decades and applied in various fields like surveillance, exploration, target localization, satellite formation flight and robotics [1].

The goal of formation control is to drive all the autonomous agents to reach and maintain a desired geometric shape. Sometimes, the whole formation is required to track a desired trajectory while maintaining the geometric shape, which is known as formation tracking. The cooperative control of the agents can be achieved through centralized or distributed schemes. The latter has obvious advantages which include

S. A. Ajwad and P. Coirault are with the Universite de Poitiers, LIAS (EA 6315), 2 rue Pierre Brousse, 86073 Poitiers, France (e-mail: syed.ali.ajwad@univ-poitiers.fr, patrick.coirault@univ-poitiers.fr).

E. Moulay is with the Université de Poitiers, XLIM (UMR CNRS 7252), 11 bd Marie et Pierre Curie, 86073 Poitiers, France (e-mail: emmanuel.moulay@univ-poitiers.fr).

M. Defoort is with the Universite Polytechnique Hauts-de-France, INSA, LAMIH (UMR CNRS 8201), Le Mont Houy, 59313 Valenciennes, France (e-mail: michael.defoort@uphf.fr).

T. Ménard is with the Université de Caen, LAC (EA 7478), 6 bd du Maréchal Juin, 14032 Caen, France (e-mail: tomas.menard@unicaen.fr). but are not limited to efficiency, scalability, robustness and reliability [2].

The research devoted to formation tracking of MAS generally falls into three categories namely, leader-follower approach [3], virtual structure method [4] and behavior based approach [5]. Another interesting approach to achieve formation tracking is through distributed consensus protocols by choosing an appropriate state deviation from the consensus value. Moreover, all the aforementioned techniques can be incorporated in consensus based formation tracking methods [6] and usually provide a more reliable and robust solution even if some of the agents are subject to a failure [7].

Many formation control techniques have been proposed in the literature, for instance [8]-[10] etc. It is commonly assumed that the states of an agent is available to its neighbors either continuously or at regular time instants. Nevertheless, this assumption is not always valid in real-world scenarios. Indeed, in practical applications, measurement and transmission of the whole state are often impossible due to the compact size of the agent or the cost. In such cases, it is necessary to estimate the unavailable states from the available information. Moreover, due to the digital nature of the computation and transmission equipment, nonuniform sampling periods are inevitable in real-world scenarios. Furthermore, to avoid clock synchronization, each agent transmits its information at asynchronous time instants. The problem of asynchronous communication in MAS has been widely discussed in the literature, e.g. [11], [12]. Asynchronous transmission is sometimes also advantageous in a sense that it could reduce the communication load on the network since each agent in MAS transmits at different time instants. Therefore, some researchers have proposed event-triggering based techniques where information among the agents is communicated in a controlled asynchronous manner [13], [14]. In fact, in event-triggered based consensus protocols, information is communicated only when a particular event occurs. However, in this type of algorithm, pre-information of the event-triggering mechanism or function is required. Furthermore, in [13], [14], it is considered that all the states are available to the agent and its neighbors. To cope with the aforementioned communication constraints, a leaderless (resp. leader-following) scheme has been proposed for second-order MAS in [15] (resp. in [16]). These control algorithms were based on continuous-discrete time observers [17] which reconstructed position and velocity in continuoustime from the discrete position data. Recently, an attempt to deal with the formation tracking problem under communication constraints has been proposed in [18]. However, in this 
paper, collision avoidance is not considered which is another important issue in the formation tracking problem. It is vital to avoid collision between the agents while they converge to the desired geometric shape. Among various techniques for collision avoidance, the Artificial Potential Field (APF) method has been widely used for MAS due to its efficiency and simplicity [19], [20].

In this article, a novel distributed formation tracking algorithm with collision avoidance is proposed for doubleintegrator MAS under communication constraints which include: (i) availability of position state only to the neighbors which means that the agent velocity and acceleration (input) are not available at all, (ii) the measured position state is transmitted at arbitrary nonuniform and irregular time instants, (iii) the sampling instants of each agent are asynchronous and completely independent from the other agents in the system and (iv) the communication topology is directed. The reference trajectory is produced by an active leader and only small portion of agents have access to the leader position. It must be noted that [16] only deals with consensus tracking problem while the current research work focuses on the design of collision-free formation tracking algorithm. To the best of the authors' knowledge, our article is the first attempt that deals with the problem of collision-free formation tracking of MAS under the above mentioned communication constraints (e.g. asynchronous and aperiodic sampled position data).

\section{Preliminaries and PROBlem Statement}

\section{A. Preliminaries}

A directed graph $\mathcal{G}$ is a pair $(\mathcal{V}, \mathcal{E})$, where $\mathcal{V}$ is a nonempty finite set of nodes and $\mathcal{E} \subseteq \mathcal{V} \times \mathcal{V}$ denotes a set of edges. An edge represents an ordered pair of distinct nodes. An edge $(i, j)$ of graph $\mathcal{G}$ means that node $j$ can receive data from node $i$. A graph has a directed spanning tree if there exists a directed path from the root to all other nodes. The adjacency matrix $\mathcal{A}=\left(a_{i j}\right)$ is an $N \times N$ matrix of graph $\mathcal{G}$ with $N$ nodes, where $a_{i j}=1$ if node $i$ can receive data from node $j$ and $a_{i j}=0$ otherwise. The Laplacian matrix $\mathcal{L} \in \mathbb{R}^{N \times N}$ is defined as $l_{i i}=\sum_{j \neq i} a_{i j}, l_{i j}=-a_{i j}$ for $i \neq j$. Let a diagonal matrix $\mathcal{B}=\operatorname{diag}\left(b_{1}, b_{2}, \ldots, b_{N}\right)$ represent the connectivity between the leader and the $N$ followers. If follower $j$ can receive information from the leader then $b_{j}=1$, otherwise $b_{j}=0$. The communication graph combining both the leader and the followers is denoted by $\overline{\mathcal{G}}$. More details on graph theory can be found in Appendix B of [21].

Assumption 1: The directed graph $\overline{\mathcal{G}}$ has a directed spanning tree with the leader as a root.

Matrix $\mathcal{H}=\mathcal{L}+\mathcal{B}$ is a nonsingular M-matrix due to Assumption 1 [22]. Furthermore, there exists a diagonal matrix $\Omega=\operatorname{diag}\left(\omega_{1}, \ldots, \omega_{N}\right)$ such that $\mathcal{H}^{T} \Omega+\Omega \mathcal{H}>0$ [23]. Let

$$
\begin{aligned}
\omega_{\max } & =\max \left\{\omega_{1}, \ldots, \omega_{N}\right\} \\
\rho & =\lambda_{\min }\left(\mathcal{H}^{T} \Omega+\Omega \mathcal{H}\right)
\end{aligned}
$$

where $\lambda_{\min }($.$) represents the smallest eigenvalue.$

Lemma 1: [16] Suppose that $v_{1}(t)$ and $v_{2}(t)$ are real valued functions verifying $\frac{d}{d t}\left(v_{1}^{2}(t)+v_{2}^{2}(t)\right) \leq-a v_{1}^{2}(t)-$ $b v_{2}^{2}(t)+c \int_{t-\delta}^{t} v_{2}^{2}(s) d s+k$ for $t \geq 0$, where $a, b, \delta>0$ and $c, k \geq 0$. There exists $\varrho>0$ and $\bar{\alpha} \geq 0$ such that if $\delta<\varrho$, then $v_{1}^{2}(t)+v_{2}^{2}(t) \leq \bar{\alpha} e^{-\sigma t}+\frac{k}{\sigma}, \forall t \geq 0$ where $\sigma$ is given by $\sigma=\frac{1}{2} \min (a, b)$.

\section{B. Problem statement}

Consider a MAS consisting of followers $1 \ldots N$ with the following second-order dynamics

$$
\dot{x}_{i}(t)=A x_{i}(t)+B u_{i}(t), \quad y_{i}(t)=C x_{i}(t)
$$

for $i=1, \ldots, N$, where $x_{i}(t)=\left[p_{i}(t)^{T}, v_{i}(t)^{T}\right]^{T}$ with $p_{i}(t)$ and $v_{i}(t) \in \mathbb{R}^{m}$ are the position and the velocity respectively while $u_{i} \in \mathbb{R}^{m}$ represents the input of agent $i$. $A=\left(\begin{array}{ll}0_{m} & I_{m} \\ 0_{m} & 0_{m}\end{array}\right), B=\left(\begin{array}{c}0_{m} \\ I_{m}\end{array}\right)$ and $C=\left(\begin{array}{ll}I_{m} & 0_{m}\end{array}\right)$ are the system input and output matrices, respectively. The dynamics of the leader is given by

$$
\dot{x}_{0}(t)=A x_{0}(t)+B u_{0}(t), \quad y_{0}(t)=C x_{0}(t)
$$

The input of the leader $u_{0}$ is independent and is not affected by other agents in the network. It can be designed to achieve any required reference trajectory for the followers. Only the following assumption is made.

Assumption 2: The input of the leader $u_{0}(t)$ is bounded by a constant $\delta_{0} \geq 0$, i.e. for all $t \geq 0$ one has $\left\|u_{0}(t)\right\| \leq \delta_{0}$.

Following are the considerations in this article: (a) each agent in the network including the leader can only measure its position $p_{i}$; (b) an agent cannot measure its velocity $v_{i}$ and its input $u_{i}$; (c) each agent transmits its position information to its neighbors at irregular time intervals, the sampling instants for data transmission of each agent are totally independent from all other agents; (d) the leader can send its position data to only a few agents in the network; (e) The communication among the neighbors is one-way and is modeled through the directed graph $\overline{\mathcal{G}}$. Let $t_{k}^{i, j}$ be the instant when agent $j$ transmits its position information to agent $i$ where $i=1, \ldots, N, j=$ $0, \ldots, N(j \neq i)$ and $k \in \mathbb{N}$ such that $0<t_{k+1}^{i, j}-t_{k}^{i, j}<\tau_{M}$ where $\tau_{M}$ is the maximum allowable sampling time. The formation shape can be specified by a desired formation vector $F=\left[f_{1, p}^{T} \ldots f_{N, p}^{T}\right]$ where $f_{i, p} \in \mathbb{R}^{m}$ is the corresponding position offset of agent $i$ with respect to the leader. It is worth noting that the formation information is communicated to the agents beforehand and is not transferred during the tracking process.

Definition 1: The formation tracking of MAS (3)(4) for a given input $u_{0}(t)$ is practically achieved if $\lim \sup _{t \rightarrow+\infty}\left\|\left(x_{i}(t)-f_{i}-x_{0}(t)\right)\right\| \leq \varepsilon$, for $i=1 \ldots N$,

where $\varepsilon>0$ is a positive constant and $f_{i}=\left[\begin{array}{ll}f_{i, p}^{T} & \mathbf{0}_{1 \times m}\end{array}\right]^{T}$. The objective of agent $i$ is to achieve and maintain the desired position in the formation pattern while avoiding collision with other agents. Let each agent have a circular safety disc around it with radius $r$. Then collision avoidance means that no intersection should occur between the agents' discs i.e. $\left\|p_{i}-p_{j}\right\|>2 r$ for all $i, j, i \neq j$ at each time instant.

\section{FORMATION TRACKING AND COLLISION AVOIDANCE PROTOCOL DESIGN}

In this section, first we propose a consensus based formation tracking algorithm by introducing position offset $f_{i, p}$ in the 
consensus law of [16]. If a proper formation matrix $F$ is chosen, the agents do not collide with each other once the desired formation is achieved. However, during the transition phase, one still needs a collision avoidance mechanism. Therefore, in the second step, we update the proposed formation tracking algorithm and introduce a collision avoidance term in it to ensure that the agents do not collide while converging to the desired formation. The stability analysis of the collision-free formation tracking algorithm becomes more complicated since the overall dynamics of the controller are changed due to the additional terms for collision avoidance. Hence, it cannot be achieved via a simple extension of the results of [16].

\section{A. Formation tracking control}

The control input for formation tracking is given by

$$
\begin{aligned}
u_{i}^{f}(t) & =-\bar{c} \phi^{2}\left[\sum_{j=1}^{N} a_{i j}\left[\hat{p}_{i, i}(t)-f_{i, p}-\hat{p}_{i, j}(t)+f_{j, p}\right]\right. \\
& \left.+b_{i}\left[\hat{p}_{i, i}(t)-f_{i, p}-\hat{p}_{i, 0}(t)\right]\right]-\bar{c} 2 \phi\left[\sum _ { j = 1 } ^ { N } a _ { i j } \left[\hat{v}_{i, i}(t)\right.\right. \\
& \left.\left.-\hat{v}_{i, j}(t)\right]+b_{i}\left[\hat{v}_{i, i}(t)(t)-\hat{v}_{i, 0}(t)\right]\right]
\end{aligned}
$$

where $\phi$ and $\bar{c}$ denote the controller gain and coupling strength respectively while $\hat{p}_{i, j}$ and $\hat{v}_{i, j}$ are the estimated position and velocity of agent $j$ by agent $i$ and are given by

$$
\begin{aligned}
\dot{\hat{x}}_{i, j}(t)= & A \hat{x}_{i, j}(t)-\theta \Delta_{\theta}^{-1} K_{o} e^{-2 \theta\left(t-\kappa_{i, j}(t)\right)}\left[\hat{p}_{i, j}\left(\kappa_{i, j}(t)\right)\right. \\
& \left.-p_{j}\left(\kappa_{i, j}(t)\right)\right]
\end{aligned}
$$

with $\hat{x}_{i, j}(t)=\left[\hat{p}_{i, j}^{T}, \hat{v}_{i, j}^{T}\right]^{T}, \Delta_{\theta}=\left(\begin{array}{cc}I_{m} & 0_{m} \\ 0_{m} & \frac{1}{\theta} I_{m}\end{array}\right), K_{o}=$ $\left[\begin{array}{ll}2 I_{m} & I_{m}\end{array}\right]^{T}$ while $\theta$ represents the observer tuning parameter. $\kappa_{i, j}(t)=\max \left\{t_{k}^{i, j} \mid t_{k}^{i, j} \leq t, k \in \mathbb{N}\right\}$ is the last instant when agent $i$ receives the position data of agent $j$. The above expression (6) represents a high-gain continuous-discrete time observer which estimates position and velocity of an agent as well as its neighbors in continuous time from discrete position data. The dynamics of the observer is nonlinear due to time-varying exponential gain. Observers with timevarying gains are specially useful for the state estimation of irregular sampled-data system [24], [25]. Therefore, the overall dynamics of the closed-loop system with controller (5) and observer (6) becomes nonlinear.

Theorem 1: Consider the MAS (3)-(4) with formation tracking protocol (5)-(6) and suppose that Assumptions 1-2 hold, then there exist $\epsilon \in(0,1)$ and $\bar{\varrho}>0$ such that if

$$
\begin{aligned}
\theta & \leq \frac{\bar{\varrho}}{\tau_{M}} \\
\bar{c} & \geq \frac{\omega_{\max }}{\rho} \\
\phi & =\epsilon \theta
\end{aligned}
$$

then

$$
\sum_{i=1}^{N}\left\|e_{i}(t)\right\| \leq \alpha e^{-\frac{\phi}{\gamma} t}+\frac{\beta \delta_{0}}{\phi}, \quad \forall t \geq 0
$$

where $e_{i}$ is the formation tracking error of agent $i$ defined as $e_{i}=x_{i}-x_{0}-f_{i}, \alpha, \gamma>0, \delta_{0}$ is the upper bound of the leader input and $\beta>0$ does not depend on $\theta, \phi, \bar{c}, \tau_{M}$ and the initial conditions of the agents and observers. $\omega_{\max }$ and $\rho$ are defined in (1) and (2) respectively.

Proof: Denoting the tracking error as $e_{i}=x_{i}-x_{0}-f_{i}$ and the estimation error $\tilde{x}_{i, j}=\hat{x}_{i, j}-x_{j}$ for $j=0 \ldots N$ and $i=1 \ldots N$, input (5) can be written as

$u_{i}^{f}=-\bar{c} K_{c} \Gamma_{\phi} \sum_{k=1}^{N} \mathcal{H}_{i k} e_{k}-\bar{c} K_{c} \Gamma_{\phi} \sum_{k=1}^{N} \mathcal{H}_{i k} \tilde{x}_{i, k}+b_{i} \bar{c} K_{c} \Gamma_{\phi} \tilde{x}_{i, 0}$

where $K_{c}=\left(\begin{array}{ll}I_{m} & 2 I_{m}\end{array}\right), \Gamma_{\phi}=\operatorname{diag}\left(\phi^{2} I_{m}, \phi I_{m}\right)$ and $\mathcal{H}_{i k}$ is $i k^{\text {th }}$ element of matix $\mathcal{H}$. The tracking error dynamics can be expressed as $\dot{e}_{i}=A e_{i}+B\left(u_{i}^{f}-u_{0}\right)$. Similarly, the estimation error dynamics can be re-written as

$\dot{\tilde{x}}_{i, j}(t)=\left(A-\theta \Delta_{\theta}^{-1} K_{o} C\right) \tilde{x}_{i, j}(t)-\theta \Delta_{\theta}^{-1} K_{o} z_{i, j}(t)-B u_{j}^{f}(t)$

where $z_{i, j}(t)=\left[e^{-2 \theta\left(t-\kappa_{i, j}(t)\right)} C \tilde{x}_{i, j}\left(\kappa_{i, j}(t)\right)-C \tilde{x}_{i, j}(t)\right]$. Considering the new coordinates $\bar{e}_{i}=\Gamma_{\phi} e_{i}$ and $\bar{x}_{i, j}=\Delta_{\theta} \tilde{x}_{i, j}$ and using the equalities, $\Delta_{\theta} A \Delta_{\theta}^{-1}=\theta A, C \Delta_{\theta}^{-1}=C$, $\Gamma_{\phi} A \Gamma_{\phi}^{-1}=\phi A, \Gamma_{\phi} B=\phi B, \Delta_{\theta} B=\frac{1}{\theta} B$ and $B^{T} \Delta_{\theta}^{-1}=$ $\theta B^{T}$, one can obtain $\dot{\bar{e}}_{i}=\phi A \bar{e}_{i}+\phi B u_{i}^{f}-\phi B u_{0}, \dot{\bar{x}}_{i, j}=$ $\theta\left(A-K_{o} C\right) \bar{x}_{i, j}-\theta K_{o} z_{i, j}-\frac{1}{\theta} B u_{j}^{f}$ and

$u_{i}^{f}=-\bar{c} K_{c}\left[\sum_{k=1}^{N} \mathcal{H}_{i k} \bar{e}_{k}-\Gamma_{\phi} \Delta_{\theta}^{-1}\left(\sum_{k=1}^{N} \mathcal{H}_{i k} \bar{x}_{i, k}+b_{i} \bar{x}_{i, 0}\right)\right]$

Denoting $\eta^{c}=\left[\bar{e}_{1}^{T} \ldots \bar{e}_{N}^{T}\right]^{T}, \eta_{i}^{o}=\left[\left(\bar{x}_{i, 1}\right)^{T} \ldots\left(\bar{x}_{i, N}\right)^{T}\right]^{T}$, $i=1 \ldots N$ and $\eta_{0}^{o}=\left[\left(\bar{x}_{1,0}\right)^{T} \ldots\left(\bar{x}_{N, 0}\right)^{T}\right]$, it can be found that $\dot{\eta}^{c}, \dot{\eta}_{i}^{o}$ and $\dot{\eta}_{0}^{o}$ are similar to ones provided in [16]. Following the procedure of [16] and Lemma 1, one can achieve inequality (10) which ends the proof.

Remark 1: Inequality (10) shows that the MAS achieves practical stability which means that the error will remain in a ball of an arbitrary small radius and centred at the origin. It is worth noting that the MAS will achieve exponential stability if leader is stationary or moving with constant velocity, i.e. leader without external input.

Remark 2: It can be seen from inequality (10) that the final error can be reduced by increasing controller parameter $\phi$. It will also increase the convergence rate. However, the controller dynamics must be slower than the observer dynamics in order to guarantee the stability of the closed-loop system which is represented by (9) with the fact that $\epsilon<1$. Moreover, (7) shows that the observer parameter $\theta$ is directly affected by the maximum sampling time $\tau_{M} . \theta$ must be chosen small enough for the case of large $\tau_{M}$.

Remark 3: The structure of the proposed observer-based algorithm shows some advantages to handle communication delays in the measurements and data packet dropouts. As each controller is using the estimated states provided by the local observer, by time stamping the measured position data, the estimation can be provided as soon as the data is received, even with a delay. Moreover, if the information packet is lost during communication, the observer could still provide the estimation if the next data is available within $\tau_{M}$ duration with respect to the last available data. 


\section{B. Collision avoidance}

For the implementation of the formation tracking controller, the safety distance between agents (i.e. $2 r$ ) should be considered while agents converge to the desired position. Indeed, without this constraint, agents could collide and be damaged. Therefore, it is of utmost importance to include some collision avoidance mechanisms in order to achieve collision-free formation tracking. In this paper, a repulsion force between agents to avoid collision has been introduced using APF. It allows agents to repel each other when the distance between them becomes smaller than a certain value. All the agents which are nearby and in the restricted vicinity of an agent are considered as obstacles. Based on the practical aspects, an ideal potential function must have the following properties: (i) the range of the potential field must be bounded. Usually, it depends on the range of obstacle sensors mounted on the agent, (ii) the value of the potential field and the corresponding repulsion must be infinity at the boundary of the obstacle and must decrease with the increase in the distance and (iii) first and second derivatives of the potential function must exist in order to have a smooth repulsion force.

Assumption 3: It is assumed that agents are equipped with proximity sensors and can detect the distance (relative position) of any non-cooperative and cooperative entities within a sensing range $R>0$.

Remark 4: Sensing capability is required to sense the presence of any other agent in the close vicinity which may lead to a collision. These sensors give the relative position of any agent within its range in the local frame and do not provide position information in the global frame. It is to note that this assumption is used for the purpose of collision avoidance only.

Let us define the following APF [26], [27]

$$
q_{i j}=\left(\min \left\{0, \frac{\left\|p_{i j}\right\|^{2}-R^{2}}{\left\|p_{i j}\right\|^{2}-4 r^{2}}\right\}\right)^{2}
$$

where $r>0$ is the radius of the safety disc around an agent and $p_{i j}=p_{i}-p_{j}$. From (11), one gets $q_{i j}>0$ if the distance between agents $i$ and $j$ is less than $R$. It is also clear that $q_{i j}$ tends to infinity if the inter-agent distance tends to $r$ and $q_{i j}=q_{j i}$. The partial derivative of the potential function is

$$
\frac{\partial q_{i j}^{T}}{\partial p_{i}}= \begin{cases}\frac{4\left(\left\|p_{i j}\right\|^{2}-R^{2}\right)\left(R^{2}-4 r^{2}\right)}{\left(\left\|p_{i j}\right\|^{2}-4 r^{2}\right)^{3}} p_{i j}^{T} & \text { if } 2 r \leq\left\|p_{i j}\right\| \leq R \\ 0 & \text { otherwise }\end{cases}
$$

and $\frac{\partial q_{i j}}{\partial p_{i}}=-\frac{\partial q_{i j}}{\partial p_{j}}=\frac{\partial q_{j i}}{\partial p_{i}}=-\frac{\partial q_{j i}}{\partial p_{j}}$.

The total repulsion force on one agent to avoid collision is

$$
u_{i}^{r}(t)=-\sum_{j=0}^{N} \frac{\partial q_{i j}^{T}}{\partial p_{i}}
$$

The overall formation controller with collision avoidance is then designed as follows

$$
u_{i}(t)=u_{i}^{f}(t)+u_{i}^{r}(t)
$$

Assumption 4: The initial configuration of the agents is outside of the detection radius of the others. It means that
$\left\|p_{i}(0)-p_{j}(0)\right\|>R$ for all $i, j, i \neq j$. Moreover, the formation shape is chosen such that the inter-agent distance in the desired formation remains greater than $R$.

Theorem 2: Consider the MAS (3)-(4) with formation tracking protocol (14) and suppose that Assumptions 1-4 hold. If conditions (7)-(9) are satisfied,

then the formation tracking is practically achieved without any inter-agent collision.

Proof: Let us define the position and velocity tracking errors as $\xi_{i}=p_{i}-f_{i, r}-p_{0}$ and $\zeta_{i}=v_{i}-v_{0}$ respectively.

Step 1: First, consider system (3) with only formation controller (5) and choose the Lyapunov function as

$$
V_{1}=\frac{1}{2} \sum_{i=1}^{N}\left[\xi_{i}^{T} \xi_{i}+\zeta_{i}^{T} \zeta_{i}\right]
$$

The time derivative of $V_{1}$ along the trajectories of system (3) is

$$
\dot{V}_{1}=\sum_{i=1}^{N}\left[\xi_{i}^{T} \dot{\xi}_{i}+\zeta_{i}^{T}\left(u_{i}^{f}-u_{0}\right)\right]
$$

Moreover, (15) can also be written as $V_{1}=\frac{1}{2} \sum_{i=1}^{N} e_{i}^{T} e_{i}=$ $\frac{1}{2} \sum_{i=1}^{N}\left\|e_{i}(t)\right\|^{2}$. By using (10), one has for all $t \geq 0$ :

$$
\begin{aligned}
\sqrt{2 V_{1}} & \leq \alpha e^{-\frac{\phi}{\gamma} t}+\frac{\beta \delta_{0}}{\phi} \\
V_{1} & \leq \frac{1}{2}\left(\alpha e^{-\frac{\phi}{\gamma} t}+\frac{\beta \delta_{0}}{\phi}\right)^{2}
\end{aligned}
$$

Step 2: Let us now consider system (3) with controller (14) which includes both formation tracking and collision avoidance. Define the following Lyapunov function:

$V_{2}=\frac{1}{2} \sum_{i=1}^{N}\left(\left[\xi_{i}^{T} \xi_{i}+\zeta_{i}^{T} \zeta_{i}\right]+\sum_{j=1}^{N} q_{i j}\left(p_{i}, p_{j}\right)\right)+\sum_{i=1}^{N} q_{i 0}\left(p_{i}, p_{0}\right)$

The last two terms in the above equation are corresponding to the potential function which are needed to guarantee the collision avoidance. The time derivative of $V_{2}$ along the trajectories of system (3) is

$$
\begin{aligned}
\dot{V}_{2}= & \sum_{i=1}^{N}\left[\xi_{i}^{T} \dot{\xi}_{i}+\zeta_{i}^{T} \dot{\zeta}_{i}\right]+\frac{1}{2} \sum_{i=1}^{N} \sum_{j=1}^{N}\left(\frac{\partial q_{i j}}{\partial p_{i}} v_{i}+\frac{\partial q_{i j}}{\partial p_{j}} v_{j}\right) \\
& +\sum_{i=1}^{N}\left(\frac{\partial q_{i 0}}{\partial p_{i}} v_{i}+\frac{\partial q_{i 0}}{\partial p_{0}} v_{0}\right) \\
= & \sum_{i=1}^{N}\left[\xi_{i}^{T} \dot{\xi}_{i}+\zeta_{i}^{T}\left(u_{i}^{f}-u_{0}\right)\right]+\sum_{i=1}^{N}\left(\frac{\partial q_{i 0}}{\partial p_{i}} v_{i}+\frac{\partial q_{i 0}}{\partial p_{0}} v_{0}\right) \\
& +\sum_{i=1}^{N} \zeta_{i}^{T} u_{i}^{r}+\frac{1}{2} \sum_{i=1}^{N} \sum_{j=1}^{N}\left(\frac{\partial q_{i j}}{\partial p_{i}} v_{i}+\frac{\partial q_{i j}}{\partial p_{j}} v_{j}\right) \\
= & \dot{V}_{1}-\sum_{i=1}^{N}\left(v_{i}-v_{0}\right)^{T} \sum_{j=o}^{N} \frac{\partial q_{i j}^{T}}{\partial p_{i}}+\sum_{i=1}^{N} \sum_{j=1}^{N} \frac{\partial q_{i j}}{\partial p_{i}} v_{i} \\
& +\sum_{i=1}^{N} \frac{\partial q_{i 0}}{\partial p_{i}}\left(v_{i}-v_{0}\right)
\end{aligned}
$$


Since $\frac{1}{2} \sum_{i=1}^{N} \sum_{j=1}^{N}\left(\frac{\partial q_{i j}}{\partial p_{i}} v_{i}+\frac{\partial q_{i j}}{\partial p_{j}} v_{j}\right)=\sum_{i=1}^{N} \sum_{j=1}^{N} \frac{\partial q_{i j}}{\partial p_{i}} v_{i}$, one obtains

$$
\dot{V}_{2}=\dot{V}_{1}+v_{0}^{T} \sum_{i=1}^{N} \sum_{j=1}^{N} \frac{\partial q_{i j}^{T}}{\partial p_{i}}
$$

Furthermore, since $\sum_{i=1}^{N} \sum_{j=1}^{N} \frac{\partial q_{i j}}{\partial p_{i}}=0$, it leads to

$$
\dot{V}_{2}=\dot{V}_{1}
$$

which implies that if the initial positions of the agents satisfy Assumption 4 , i.e. $\frac{\partial q_{i j}}{\partial p_{i}}=0$ at $t=0$ for $i=1 \ldots N, j=$ $0 \ldots N$, consequently

$$
V_{1}(0)=V_{2}(0)
$$

then

$$
V_{2}=V_{1} \leq \frac{1}{2}\left(\alpha e^{-\frac{\phi}{\gamma} t}+\frac{\beta \delta_{0}}{\phi}\right)^{2}, \quad \forall t \geq 0 .
$$

Hence, the MAS achieves the desired formation with controller (14) in practical sense. Also, from the the structure of (12) and (13), one has $\lim _{\left\|p_{i j}\right\| \rightarrow r} q_{i j}=\infty$ and $\lim _{\left\|p_{i j}\right\| \rightarrow r} \frac{\partial q_{i j}}{\partial p_{i}}=\infty$

for $\forall i \neq j$. Therefore, it can be concluded that collisions are avoided.

\section{Simulation RESULTS}

For simulation purposes, the considered network consists of four followers labelled from 1 to 4 and a leader labelled as 0 . The directed communication topology among the agents is shown in Fig. 1. One can note that the leader only sends

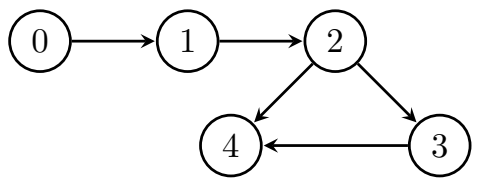

Fig. 1: Communication topology

its position data at random intervals to follower 1 while follower 4 can receive information from both followers 2 and 3 at irregular and asynchronous sampling times. The observer and controller gains are chosen as $\theta=10$ and $\phi=0.6$ respectively. The detection region $R=2$ while the safety region $r=0.25$ for each agent. The simulations are performed for 2-dimensional space which means $m=2$. The initial conditions are chosen such that Assumption 4 is verified. The desired formation is chosen to produce a square geometric shape around the leader. The corresponding position offsets for the followers are $f_{1, p}=[6,6]^{T}, f_{2, p}=[6,-6]^{T}$, $f_{3, p}=[-6,6]^{T}$ and $f_{4, p}=[-6,-6]^{T}$. For the first scenario, the leader is kept stationary at position coordinates $(0,0)$ while the followers reach and maintain the desired square shape around it. Fig. 2 illustrates the formation producing result and the inter-agent distances. The inter-agent distance clearly shows that the agents do not collide during the formation producing. Fig 3 explains collision avoidance mechanism for follower 1 . The distance between follower 1 and the other agents in the network is shown in Fig 3a while Fig 3b shows the control input. It can be seen that the repulsion term in

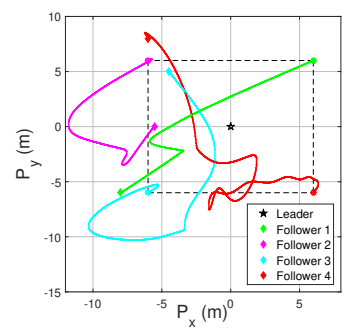

(a)

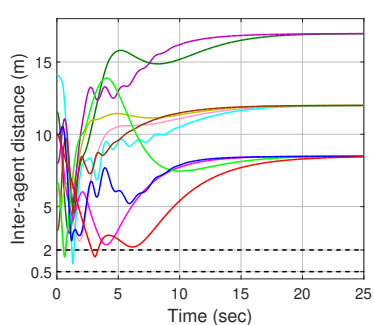

(b)
Fig. 2: Square formation with static leader: (a) Formation tracking with $\diamond=$ initial state and $o=$ final state - (b) Interagent distance

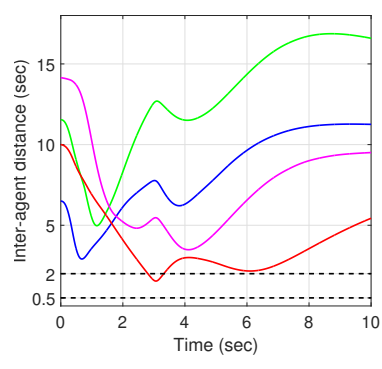

(a)

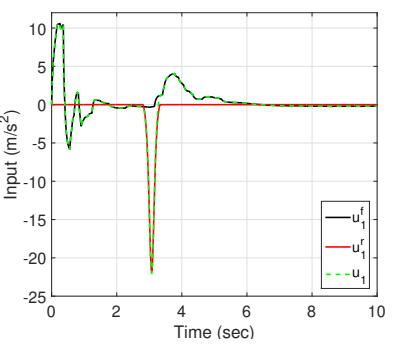

(b)
Fig. 3: Follower 1: (a) Distance with other agents - (b) Control input

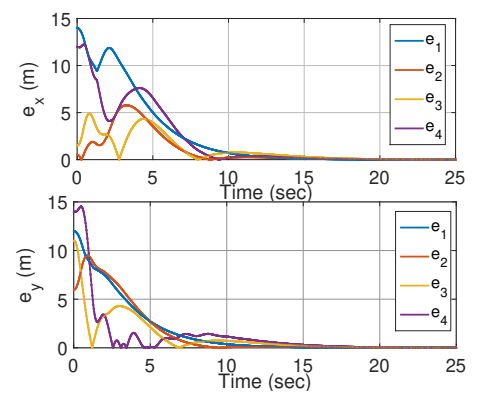

Fig. 4: Tracking error for square formation with static leader

the controller activates when the distance between agents is less than $R=2$ otherwise remains zero. For the second scenario, the leader is moving with constant acceleration i.e. $u_{x_{0}}=0.03$ and $u_{y_{0}}(0)=0.02$. Since the leader moves with constant acceleration, only practical stability is achieved as described in Remark 1. The corresponding results are depicted in Fig. 5 and Fig. 6. An example of the sampling instants for data transmission between the agents is shown in Fig. 7. In all the scenarios, the MAS achieves desired formation without any collision between agents.

\section{CONCLUSION}

The formation tracking problem with collision avoidance has been studied for MAS under communication constraints. It has been considered that the agents can only transmit their position states to the neighbors at nonuniform and asynchronous 


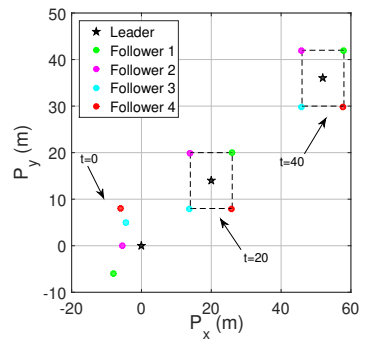

(a)

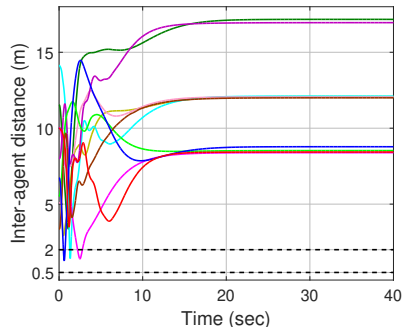

(b)
Fig. 5: Square formation with constant leader acceleration input: (a) Formation tracking - (b) Inter-agent distances
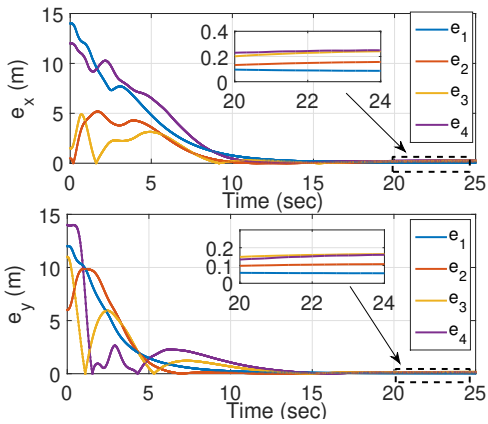

Fig. 6: Tracking error for square formation with constant leader acceleration

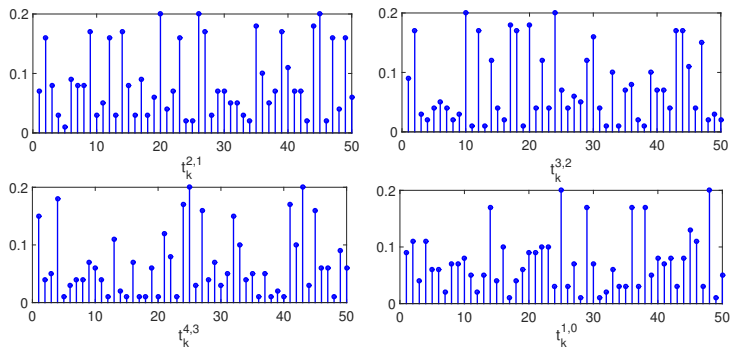

Fig. 7: Sampling time for data transmission between agents

sampling instants. Using continuous-discrete time observers and potential function based mechanism, an output feedback formation controller has been derived. The study of formation tracking consensus of nonlinear MAS under communication constraints along with noise is considered as future work.

\section{REFERENCES}

[1] Y. Liu and Y. Jia, "An iterative learning approach to formation control of multi-agent systems," Systems \& Control Letters, vol. 61, no. 1, pp. 148-154, 2012

[2] Y. Zhao, Z. Duan, G. Wen, and Y. Zhang, "Distributed finite-time tracking control for multi-agent systems: an observer-based approach," Systems \& Control Letters, vol. 62, no. 1, pp. 22-28, 2013.

[3] J. Shao, G. Xie, and L. Wang, "Leader-following formation control of multiple mobile vehicles," IET Control Theory \& Applications, vol. 1, no. 2, pp. 545-552, 2007.

[4] Q. Qin, T.-S. Li, C. Liu, C. P. Chen, and M. Han, "Virtual structure formation control via sliding mode control and neural networks," in International Symposium on Neural Networks. Springer, 2017, pp. 101-108.
[5] S. Nag and L. Summerer, "Behaviour based, autonomous and distributed scatter manoeuvres for satellite swarms," Acta Astronautica, vol. 82 no. 1, pp. 95-109, 2013.

[6] W. Ren, "Consensus strategies for cooperative control of vehicle formations," IET Control Theory \& Applications, vol. 1, no. 2, pp. 505-512, 2007.

[7] T. A. Dierks and J. Sarangapani, "Neural network control of mobile robot formations using rise feedback," IEEE Transactions on System, Man and Cybernetics: Part B, vol. 39, no. 2, pp. 332-347, 2009.

[8] M. C. De Gennaro and A. Jadbabaie, "Formation control for a cooperative multi-agent system using decentralized navigation functions," in American Control Conference. IEEE, 2006, pp. 1346-1351.

[9] L. Dou, C. Yang, D. Wang, B. Tian, and Q. Zong, "Distributed finite-time formation control for multiple quadrotors via local communications," International Journal of Robust and Nonlinear Control, vol. 29, no. 16, pp. 5588-5608, 2019

[10] H. Xiao, Z. Li, and C. P. Chen, "Formation control of leader-follower mobile robots systems using model predictive control based on neuraldynamic optimization," IEEE Transactions on Industrial Electronics, vol. 63 , no. 9 , pp. 5752-5762, 2016.

[11] J. Zhan and X. Li, "Asynchronous consensus of multiple doubleintegrator agents with arbitrary sampling intervals and communication delays," IEEE Transactions on Circuits and Systems I: Regular Papers, vol. 62, no. 9, pp. 2301-2311, 2015.

[12] W. Liu and J. Huang, "Leader-following consensus for linear multi-agent systems via asynchronous sampled-data control," IEEE Transactions on Automatic Control, vol. 65, no. 7, pp. 3215-3222, 2019.

[13] E. Garcia, Y. Cao, and D. W. Casbeer, "Periodic event-triggered synchronization of linear multi-agent systems with communication delays," IEEE Transactions on Automatic Control, vol. 62, no. 1, pp. 366-371, 2016.

[14] — " "Decentralised event-triggered consensus of double integrator multi-agent systems with packet losses and communication delays," IET Control Theory \& Applications, vol. 10, no. 15, pp. 1835-1843, 2016.

[15] T. Ménard, E. Moulay, P. Coirault, and M. Defoort, "Observer-based consensus for second-order multi-agent systems with arbitrary asynchronous and aperiodic sampling periods," Automatica, vol. 99, pp. 237$245,2019$.

[16] S. A. Ajwad, T. Ménard, E. Moulay, M. Defoort, and P. Coirault, "Observer based leader-following consensus of second-order multi-agent systems with nonuniform sampled position data," Journal of the Franklin Institute, vol. 356, no. 16, pp. 10031-10057, 2019.

[17] M. Farza, M. M'Saad, M. L. Fall, E. Pigeon, O. Gehan, and K. Busawon, "Continuous-discrete time observers for a class of mimo nonlinear systems," IEEE Transactions on Automatic Control, vol. 59, no. 4, pp. 1060-1065, 2014.

[18] S. A. Ajwad, E. Moulay, M. Defoort, T. Ménard, and P. Coirault, "Output-feedback formation tracking of second-order multiagentsystems with asynchronous variable sampled data," in IEEE Control and Decision Conference (CDC). IEEE, 2019, pp. 4483-4488.

[19] O. Khatib, "Real-time obstacle avoidance for manipulators and mobile robots," in Autonomous robot vehicles. Springer, 1986, pp. 396-404.

[20] Y. Cao and W. Ren, "Distributed coordinated tracking with reduced interaction via a variable structure approach," IEEE Transactions on Automatic Control, vol. 57, no. 1, pp. 33-48, 2011.

[21] W. Ren and R. W. Beard, Distributed consensus in multi-vehicle cooperative control. Springer, 2008, vol. 27, no. 2.

[22] Q. Song, F. Liu, J. Cao, and W. Yu, "Pinning-controllability analysis of complex networks: an m-matrix approach," IEEE Transactions on Circuits and Systems I: Regular Papers, vol. 59, no. 11, pp. 2692-2701, 2012.

[23] H. Zhang, Z. Li, Z. Qu, and F. L. Lewis, "On constructing lyapunov functions for multi-agent systems," Automatica, vol. 58, pp. 39-42, 2015.

[24] M. Farza, M. M'Saad, M. L. Fall, E. Pigeon, O. Gehan, and K. Busawon, "Continuous-discrete time observers for a class of mimo nonlinear systems," IEEE Transactions on Automatic Control, vol. 59, no. 4, pp. 1060-1065, 2013.

[25] T. Ahmed-Ali, E. Fridman, F. Giri, L. Burlion, and F. LamnabhiLagarrigue, "Using exponential time-varying gains for sampled-data stabilization and estimation," Automatica, vol. 67, pp. 244-251, 2016.

[26] Y.-H. Chang, C.-L. Chen, W.-S. Chan, H.-W. Lin, and C.-W. Chang, "Fuzzy formation control and collision avoidance for multiagent systems," Mathematical Problems in Engineering, vol. 2013, Art. no. 908180, 2013.

[27] Y. Xia, X. Na, Z. Sun, and J. Chen, "Formation control and collision avoidance for multi-agent systems based on position estimation," ISA Transactions, vol. 61, pp. 287-296, 2016. 